\title{
In situ HR-TEM characterizations on individual carbon nanotubes during its manipulation, deformation and growth
}

Chuanhong Jin, ${ }^{*}, * *$ Kazu Suenaga, ${ }^{*}$ Sumio Iijima*,**

* Nanotube Research Center, National Institute of Advanced Industrial Science and Technology (AIST), Tsukuba 305-8565, Japan

** Department of Materials Science and Engineering, Meijo University, Tenpaku-ku, Nagoya 4688502 , Japan

Investigating the physical and chemical properties of individual nanostructures has long been endeavored with the atomic configurations. Although the measurements of the structural and electronic transport properties of carbon nanotubes were previous preformed in TEM, the resolution of the image and the stability of the specimen holder were relative poor. In situ HR-TEM studies with a higher spatial resolution have been just made possible by the advancement of the electron optics in TEM based on the aberration correctors (CEOS) and the development of more stable specimen holder (Nanofactory). In this talk, we will present a few examples of the in situ HR-TEM studies of carbon nanotubes: the nanotube plumbing [1], formation and migration of individual atomic defects (large vacancies) at the elevated temperatures [2], the catalytic enlargement of fullerenes [3], catalyst-free inner growth of single-wall carbon nanotubes [4], the growth mechanism of carbon onion [5], as well as the experimental demonstration of lip-lip interactions in multi-wall carbon nanotubes [6].

References

[1] C. H. Jin et al., Nature Nanotech. 3 (2008) 17.

[2] C. H. Jin et al., ACS NANO 2 (2008) 1275.

[3] C. H. Jin et al., Phys. Rev. Lett.101 (2008) 176102.

[4] C. H. Jin et al., Nano Res. 1 (2008) 434.

[5] C. H. Jin et al., submitted work, (2009).

[5] C. H. Jin et al., Nano Lett. 8 (2008) 1127.

[6] The work on microscopy was partly supported by CREST and Grant-in-aid for Scientific Research from MEXT (19054017). C. H. J. thanks the JSPS and the International Balzan Foundation for the postdoctoral fellowships through AIST and Meijo University. 


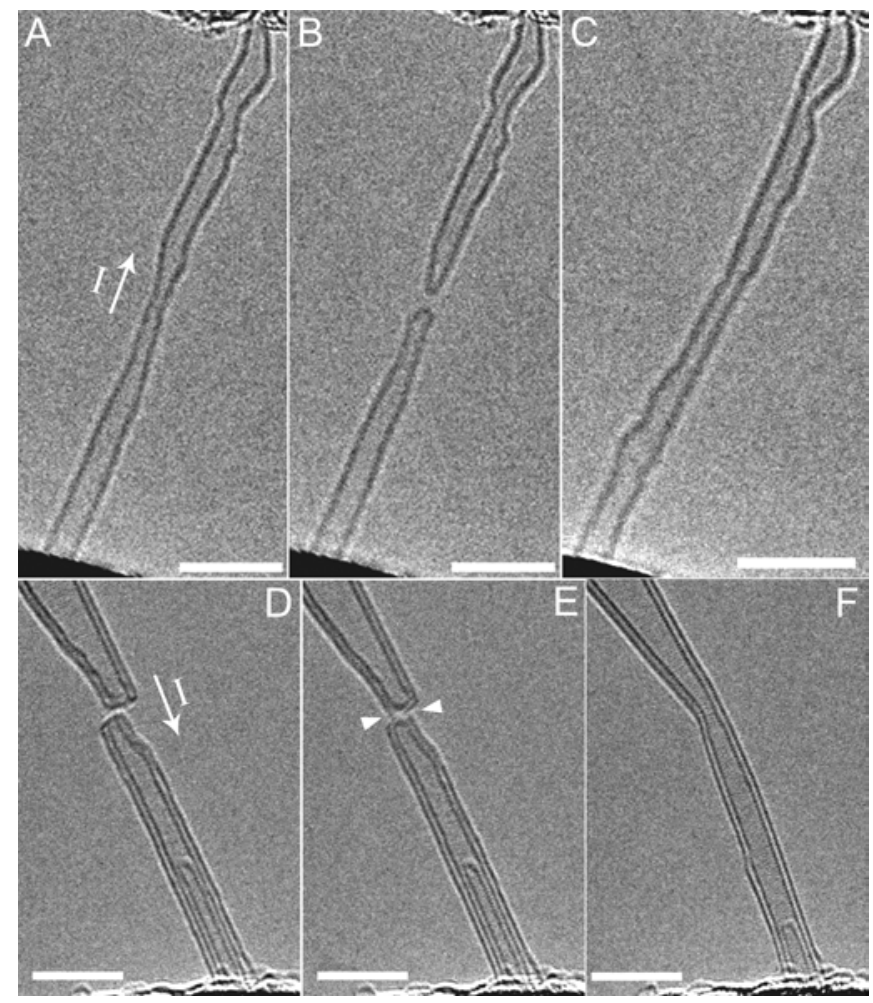

FIG. 1. Two examples showing the seamlessly joining of two SWNTs (A-C) and DWNTs (D-F) which have nearly the same diameter and chirality. Scale bar $=5 \mathrm{~nm}$.

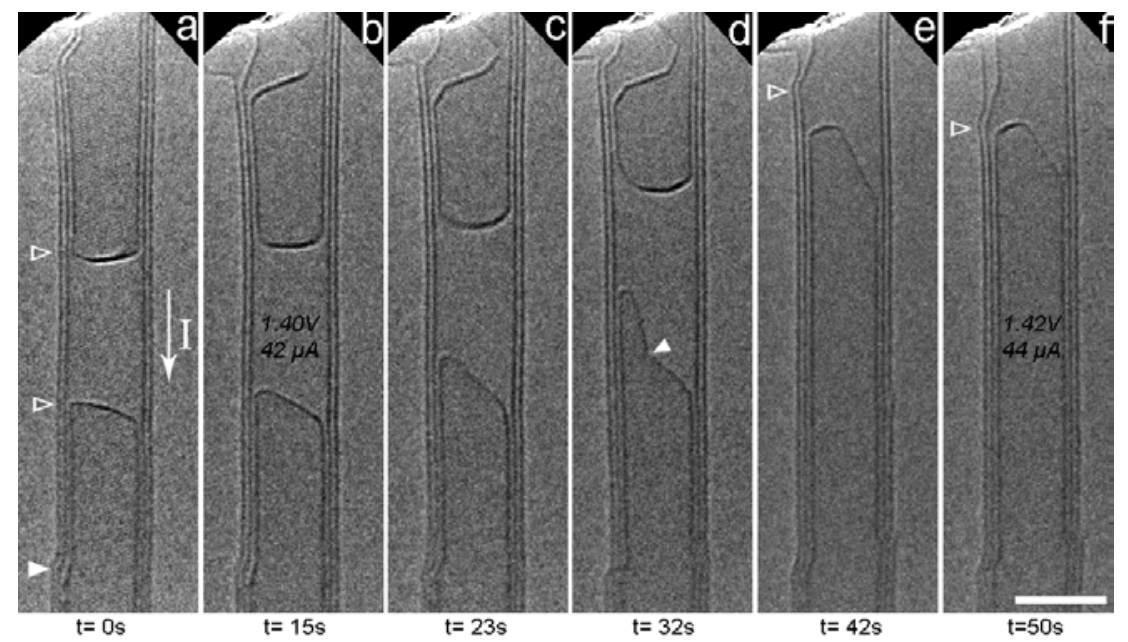

FIG. 2. A time sequential TEM image for the catalyst-free growth of SWNT inside a DWNT. Scale bar $=5 \mathrm{~nm}$. 